\title{
Formalizing Propagation of Priorities in Reo, Using Eight Colors
}

Citation for published version (APA):

Jongmans, S-S. (2018). Formalizing Propagation of Priorities in Reo, Using Eight Colors. In F. de Boer, M. Bonsangue, \& J. Rutten (Eds.), It's All About Coordination: Essays to Celebrate the Lifelong Scientific Achievements of Farhad Arbab (pp. 122-138). Springer.

Document status and date:

Published: 01/01/2018

Document Version:

Peer reviewed version

Document license:

CC BY-ND

Please check the document version of this publication:

- A submitted manuscript is the version of the article upon submission and before peer-review. There can be important differences between the submitted version and the official published version of record. People interested in the research are advised to contact the author for the final version of the publication, or visit the DOI to the publisher's website.

- The final author version and the galley proof are versions of the publication after peer review.

- The final published version features the final layout of the paper including the volume, issue and page numbers.

Link to publication

\section{General rights}

Copyright and moral rights for the publications made accessible in the public portal are retained by the authors and/or other copyright owners and it is a condition of accessing publications that users recognise and abide by the legal requirements associated with these rights.

- Users may download and print one copy of any publication from the public portal for the purpose of private study or research.

- You may not further distribute the material or use it for any profit-making activity or commercial gain

- You may freely distribute the URL identifying the publication in the public portal.

If the publication is distributed under the terms of Article 25fa of the Dutch Copyright Act, indicated by the "Taverne" license above, please follow below link for the End User Agreement:

https://www.ou.nl/taverne-agreement

Take down policy

If you believe that this document breaches copyright please contact us at:

pure-support@ou.nl

providing details and we will investigate your claim.

Downloaded from https://research.ou.nl/ on date: 26 Apr. 2023 


\title{
Formalizing Propagation of Priorities in Reo, using Eight Colors
}

\author{
Sung-Shik Jongmans ${ }^{1,2}$ \\ 1 Department of Computer Science, Open University, the Netherlands \\ 2 Department of Computing, Imperial College London, United Kingdom \\ ssj@ou.nl
}

\begin{abstract}
Reo is a language for programming of coordination protocols among concurrent processes. Central to Reo are connectors: programmable synchronization/communication mediums used by processes to exchange data. Every connector runs at a clock; at every tick, it enacts an enabled synchronization/communication among processes.

Connectors may prioritize certain synchronizations/communications over others. "Passive" connectors use their priorities only at clock ticks, to decide which enabled synchronization/communication to enact. "Active" connectors, in contrast, use their priorities also between clock ticks, to influence which synchronizations/communications become enabled; they are said to "propagate their priorities".

This paper addresses the problem of formalizing propagation of priorities in Reo. Specifically, this paper presents a new instantiation of the connector coloring framework, using eight colors. The resulting formalization of propagation of priorities is evaluated by proving several desirable behavioral equalities.
\end{abstract}

\section{Foreword}

This paper addresses, perhaps, the oldest open problem in the Reo community.

The problem came to my attention for the first time in May 2011, six months into my $\mathrm{PhD}$ project. Perhaps - nay, surely! - I should have walked away from it; oh, the time that would have saved me... But, the problem was too tempting to resist. Farhad, Kasper, and I worked on solutions intermittently over the past years. Many times, I thought we had solved it; equally many times, we had not.

I promised Farhad more than once to end our suffering (my choice of words), by formalizing propagation of priorities in the connector coloring framework, using $k>3$ colors. I never quite succeeded. This seems the perfect occasion to finally, half a decade down the road, fulfill that promise. Well, to some extent.

\section{Introduction}

Context. Reo is a language for programming of coordination protocols among concurrent processes. Central to Reo are connectors: programmable synchronization/communication mediums used by processes to exchange data, by invoking 
write and take operations. Every connector runs at a clock; at every tick, it enacts an enabled synchronization/communication among processes, based on the operations those processes have performed.

To send data, a process can invoke a write operation on the interface of a connector; to receive, it can invoke a take operation. Both writes and takes are blocking: after a process has invoked write or take, it immediately suspends, its operation becomes pending, and it resumes only after its operation has been resolved by the connector. To resolve a pending write, a connector performs a reciprocal take; to resolve a pending take, it performs a reciprocal write.

As connectors fully control resolution of pending operations, only connectors decide when (synchronization) and whereto/wherefrom (communication) data flow. In this way, connectors coordinate the synchronization/communication among processes.

Problem. Connectors may prioritize certain synchronizations/communications over others. "Passive" connectors use their priorities only at clock ticks, to decide which enabled synchronization/communication to enact. "Active" connectors, in contrast, use their priorities also between clock ticks, to influence which synchronizations/communications become enabled; they are said to "propagate their priorities".

Imagine, for instance, a connector $C$ among processes $P_{1}, P_{2}$, and $P_{3}$. Imagine, moreover, that at every clock tick, $C$ can enact either a data-flow from $P_{1}$ to $P_{3}$ with high priority (enabled only if $P_{1}$ and $P_{3}$ invoked write and take), or a data-flow from $P_{2}$ to $P_{3}$ with low priority (enabled only if $P_{2}$ and $P_{3}$ invoked write and take). If $C$ is passive, it quietly awaits the next clock tick, checks which operations are pending to determine which data-flows are enabled (if any), chooses and enacts the one with the highest priority, and quietly awaits the next clock tick. If $C$ is active, in contrast, it requests $P_{1}$ to invoke write (and $P_{3}$ to invoke take) before the next clock tick, thereby enabling $C$ to choose and enact the high priority data-flow from $P_{1}$ to $P_{3}$ at the next clock tick.

Contribution. Existing formalizations of Reo do not support modeling of connectors that propagate priorities. This paper presents such a formalization.

Section 2 establishes terminology and definitions. The section is terse; more gentle introductions to Reo Arb04Arb11 and the connector coloring framework [CCA07/Cos10] appear elsewhere. Section 3 details the problem of formalizing propagation of priorities. Section 4 presents a solution in the connector coloring framework, using eight colors. Section 5 contains an evaluation of this solution, in terms of behavioral equalities. Section 6 concludes this paper with a discussion. Appendix A contains definitions. Proofs appear in a technical report Jon18. 


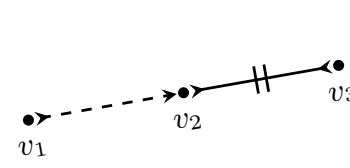

(a)

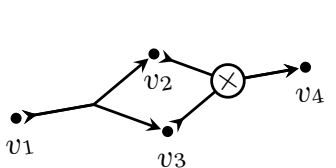

(b)

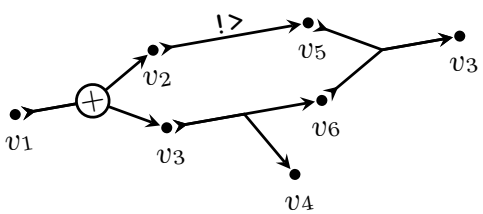

(c)

Fig. 1: Examples of connector syntax

\section{Preliminaries}

Syntax. Structurally, a connector in Reo is a (directed hyper)graph of vertices and (nonempty, directed hyper)edges ${ }^{3}$ Every edge is labeled with a type, shortly used to define the semantics of a connector. Figure 1 shows examples.

A vertex of a connector is external if it is the source of exactly one edge, or the target of exactly one edge; otherwise, it is internal. Processes perform write and take operations on external vertices, which thus consistute the interface.

A connector is primitive, if it has exactly one edge; otherwise, it is compound. Figure 2 first column, shows the name and syntax of common primitives.

A connector is well-formed, if (i) it has at least one edge, and (ii) if each of its vertices is the source of at most one edge, the target of at most one edge, and the source or target of at least one edge.

The structural composition of two connectors, denoted by operator $\bowtie$, is the graph consisting of the union of the sets of vertices, and the union of the sets of edges; it is a partial operation, to preserve well-formedness. Moreover, structural composition is associative and commutative.

A vertex is shared between two connectors, if it is an external vertex of both.

Informal semantics. Behaviorally (informal), a connector in Reo is a set of dataflows between vertices, along edges, endowed with a partial order of priorities 4

A vertex is active in a data-flow, if data passes through it; otherwise, it is passive. Every vertex participates either actively or passively in each of its connector's data-flows. Idling is the degenerate data-flow in which every vertex participates passively. A data-flow of a connector is enabled, if every external vertex that actively participates in the data-flow has a pending write or take; idling is always enabled, vacuously.

A connector runs on a clock; at every tick, it enacts one of its enabled dataflows. If multiple data-flows are enabled, it nondeterministically selects an ordertheoretically maximal one among them. Figure 2, second column, shows the informal semantics of common primitives; "prioritizes $(n)$ over $(m)$ " means " $(n)$ is greater than $(m)$ ".

\footnotetext{
${ }^{3}$ Binary edges are usually called channels; maximal sets of adjacent ternary edges are usually called nodes Arb04 Arb11.

${ }^{4}$ For simplicity, and because it is a concern orthogonal to formalizing priorities, I consider only stateless connectors in this paper.
} 


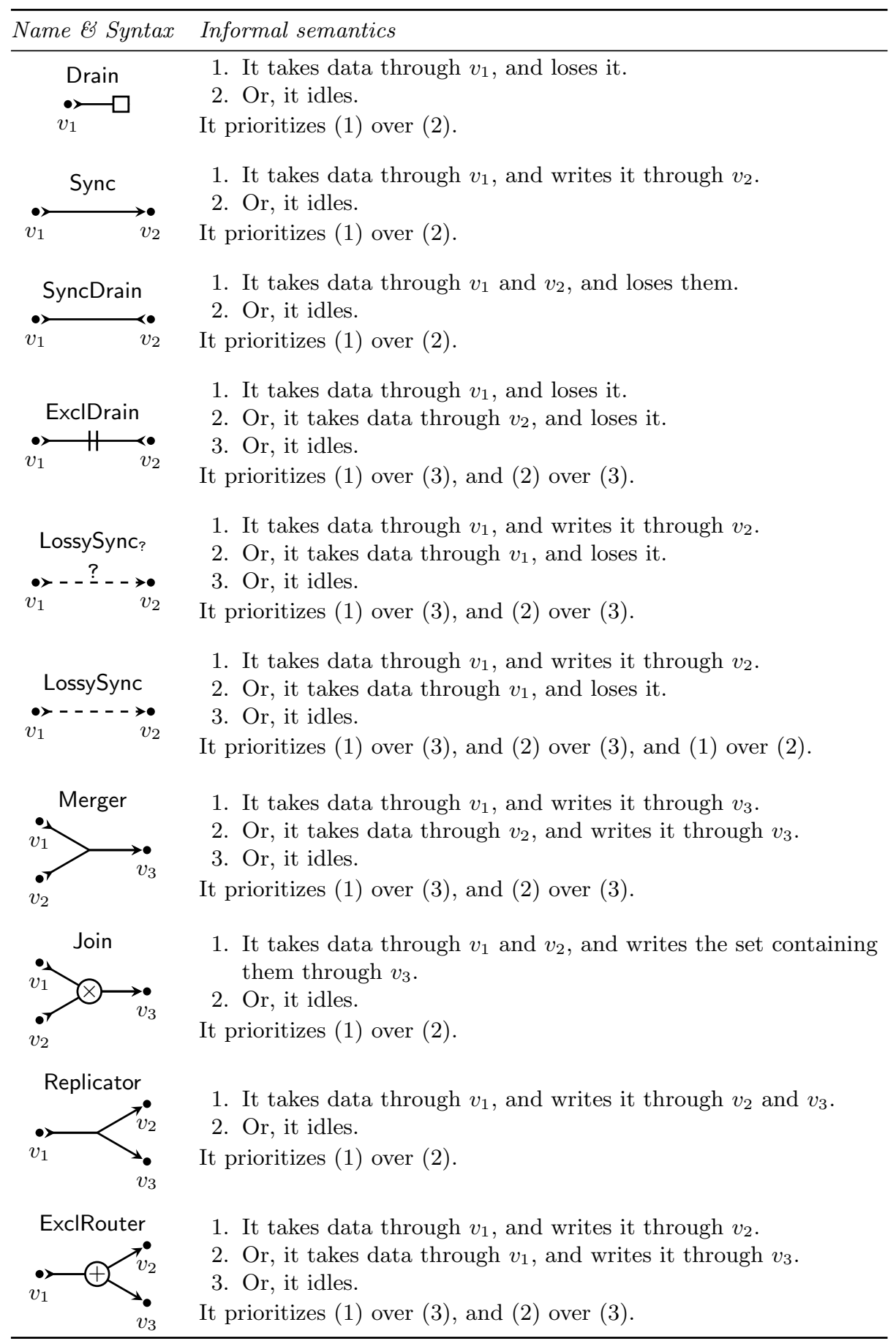

Fig. 2: Name, syntax, and informal semantics of common primitives 
$(1,1)$ It takes data through $v_{1}$, writes/takes it through $v_{2}$, and loses it.

$(2,2)$ Or, it takes data through $v_{1}$ and $v_{3}$, and loses it.

$(2,3)$ Or, it takes data through $v_{1}$ and loses it.

$(3,2)$ Or, it takes data through $v_{3}$ and loses it.

$(3,3)$ Or, it idles.

It prioritizes $(1,1)$ over $(3,3)$, and $(1,1)$ over $(2,3)$, and $(2,2)$ over $(3,3)$, and $(2,3)$ over $(3,3)$, and $(3,2)$ over $(3,3)$.

Fig. 3: Informal semantics of Fig. 1a

A data-flow through one connector is consistent with a data-flow through another connector, if each of their shared vertices is either active or passive in both data-flows. This ensures data can flow between connectors, through their shared vertices. The behavioral composition of two connectors is the set consisting of the pairs of consistent data-flows, endowed with their product order. Every global data-flow through a compound connector, thus, is the concatenation of local data-flows.

For instance, the connector in Fig. 1a is composed of LossySync and ExclDrain in Fig. 2. As these connectors both have three local data-flows, the compound has at most nine global data-flows. Figure 3 shows which of those data-flows are consistent; $(n, m)$ means "the pair consisting of $(n)$ of LossySync and $(m)$ of ExclDrain". As the compound prioritizes $(1,1)$ over $(2,3)$, and because $(1,1)$ and $(2,3)$ are always enabled together, it never enacts $(2,3)$.

Formal semantics. Behaviorally (formal), in the connector coloring framework, a connector is a set of total functions, called colorings, from vertices to natural numbers, called colors CCA07|Cos10|JKA11/CP12]. Every coloring models a data-flow; every color models the activeness/passiveness of a vertex in a dataflow. Depending on the number of colors the framework is instantiated with, different levels of activeness/passiveness can be distinguished, to lesser or greater expressiveness. In particular, colors can be used to model priorities, as an alternative to endowing sets of colorings with partial orders (exemplified shortly).

Two colorings are consistent if they map the vertices in the intersection of their domains to the same colors. The behavioral composition of two connectors, denoted by operator $\bowtie$, is the set consisting of the unions of their consistent colorings. As such, behavioral composition in the connector coloring framework straightforwardly models concatenation of consistent data-flows 5 Behavioral composition is associative and commutative.

The structure and behavior of a connector are related through a denotation function $\llbracket \cdot \rrbracket$ : it consumes as input a connector structure (graph) and produces as output a connector behavior (set of colorings), by decomposing the connector into primitives, looking up the local behavior of every primitive in a predefined type-indexed table, and composing the local behaviors into a global one.

${ }^{5}$ The composition operator can be extended with the flip-rule [CCA07/Cos10, to reduce sets of colorings. I do not pursue this in this paper. 


\begin{tabular}{|c|c|c|}
\hline$\#$ & $\stackrel{\bullet}{\longrightarrow}$ & Meaning \\
\hline 0 & $-\ldots$ & Passive \\
\hline 1 & $\longrightarrow$ & Active \\
\hline 2 & $\cdot-\triangleright-\cdot$ & Passive, for no write \\
\hline 3 & $\cdot-\triangleleft-\cdot$ & Passive, for no take \\
\hline 4 & $\rightarrow=$ & Active; metadata-flow downstream (to propagate priorities) \\
\hline 5 & $-\leftarrow$ & Active; metadata-flow upstream (to propagate priorities) \\
\hline 6 & $\Rightarrow \leftrightarrow$ & Active; metadata-flows downstream + upstream (to propagate priorities) \\
\hline 7 & $\cdot-\bowtie-\cdot$ & Passive, for no write, for conflicting propagated priorities upstream \\
\hline 8 & $\cdot-\triangleleft-\cdot$ & Passive, for no take, for conflicting propagated priorities downstream \\
\hline
\end{tabular}

Fig. 4: Colors

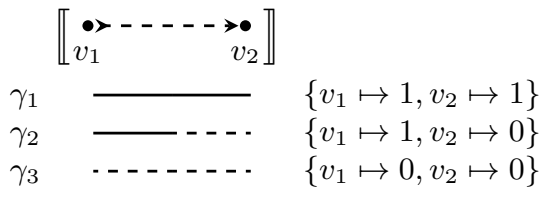

(a) LossySync

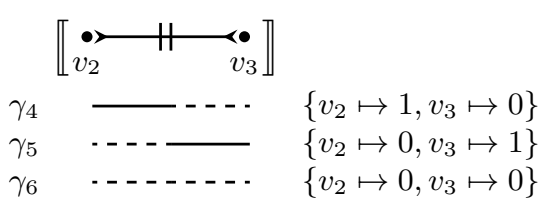

(b) ExclDrain

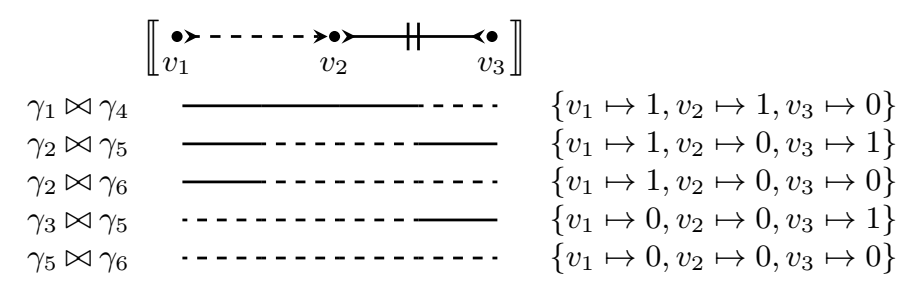

(c) Figure $1 \mathrm{a}$

Fig. 5: Examples of two-color semantics

To exemplify the connector coloring framework, Fig. 4 shows nine colors. Colors $0,1,2,3$ already exist in the literature; colors $4,5,6,7,8$ are new. The following lists summarizes three existing instantiations of the framework:

- With two colors CCA07/Cos10, $\{0,1\}$, one can model data-flows, but not priorities. Figure 5 shows examples. As the figure shows, colorings can be represented both textually and graphically (using the notation in Fig. 4). Figure 5a shows the behavior of LossySync. Coloring $\gamma_{1}$ models a data-flow from $v_{1}$ to $v_{2}$ (both vertices are active); coloring $\gamma_{2}$ models the loss of data taken through $v_{1}$ (only $v_{1}$ is active); coloring $\gamma_{3}$ models idling. Figure $5 \mathrm{~b}$ and $5 \mathrm{c}$ can be explained similarly. The colorings in Fig. 5 model exactly, oneto-one, the data-flows in Figs. 2 and 3 . However, priorities are not modeled.

- With three colors [CA07|Cos10], $\{1,2,3\}$, one can model both data-flows and priorities. Specifically, color 0 is refined into colors 2,3, to model not only that a vertex is passive, but also why. Figure 6 shows examples. I write 


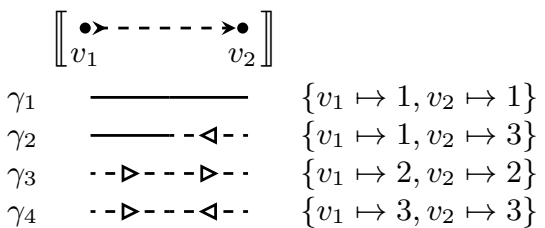

(a) LossySync

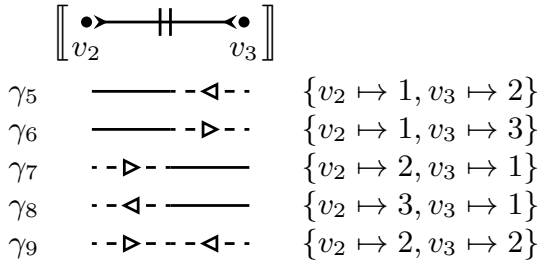

(b) ExclDrain

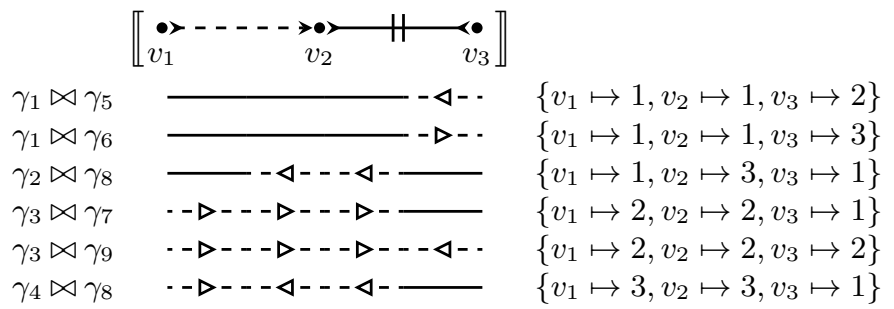

(c) Figure $1 \mathrm{a}$

Fig. 6: Examples of three-color semantics

"the environment can write/take through $v$ " to mean that either a write/ take is pending on $v$ (if the environment at $v$ is a process) or a data-flow can be concatenated at $v$ (if the environment at $v$ is another connector).

The expressive power of the three-color semantics is best exemplified with LossySync, as follows. Coloring $\gamma_{2}$ in Fig. 5a and coloring $\gamma_{2}$ in Fig. 6a both model the loss of data taken through $v_{1}$. However, $\gamma_{2}$ in Fig. 6a additionally models that this data-flow can be chosen/enacted only if no take can be resolved at $v_{2}$. As $v_{2}$ is a target vertex of LossySync (i.e., LossySync can only write through $v_{2}$ ), this happens only if the environment cannot take through $v_{2}$. Thus, if the environment can write through $v_{1}$, but not take through $v_{2}$, LossySync can lose $\left(\gamma_{2}\right)$. But, if the environment can both write and take, LossySync must choose to not-lose $\left(\gamma_{1}\right)$ instead of to lose $\left(\gamma_{2}\right)$, just as its informal semantics demands (Fig 2).

The three-color semantics of LossySync? is the same as the three-color semantics of LossySync, plus coloring $\gamma_{2}^{\prime}=\left\{v_{1} \mapsto 1, v_{2} \mapsto 2\right\}$. This extra coloring models the loss of data taken through $v_{1}$, just as $\gamma_{2}$ in Fig. 6a. However, $\gamma_{2}^{\prime}$ additionally models that this data-flow can be chosen/enacted only if no write can be resolved at $v_{2}$. As $v_{2}$ is a target vertex of LossySync? (i.e., the environment can only take through $v_{2}$ ), this happens only if LossySync? cannot write through $v_{2}$. This is a condition that LossySync? always can satisfy (independent of the environment). Thus, if the environment can both write and take, LossySync? nondeterministically chooses between not-losing $\left(\gamma_{1}\right)$ and losing $\left(\gamma_{2}^{\prime}\right)$; in the former case, it writes through $v_{2}$, while in the latter case, it does not. Thus, $\gamma_{2}^{\prime}$ is the three-color equivalent of $\gamma_{2}$ in Fig. 5a. 


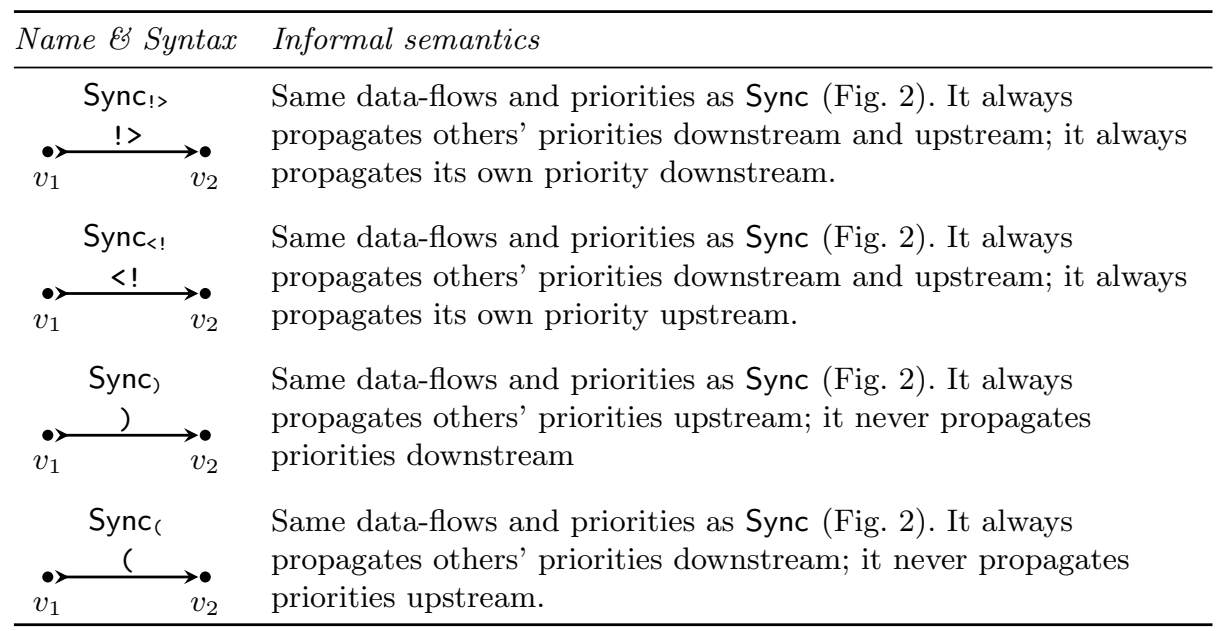

Fig. 7: Name, syntax, and informal semantics of priority primitives

LossySync and LossySync? illustrate that by carefully modeling why vertices are passive, using colors 2, 3, priorities may emerge. Graphically, the triangle markings always point away from the root cause for passiveness. For instance, in coloring $\gamma_{3} \bowtie \gamma_{7}$, vertex $v_{2}$ is passive, because there is no write on $v_{2}$ (cause), because the environment cannot write through $v_{1}$ (root cause).

- With four colors CP12, $\{0,1,2,3\}$, one can model data-flows, priorities, and partiality. The latter is useful to allow parts of a connector to skip clock ticks; this is subtly different from idling, and particularly useful in distributed connector implementations. The details do not matter in this paper.

\section{Problem}

Informally, propagation of priorities entails the following:

If a connector propagates the priority of a "superior" data-flow over an "inferior" one into the environment, it enacts the inferior data-flow only if: (i) another connector simultaneously propagates a priority into the environment, and (ii) the environment can facilitate only one of the two priorities - they are conflicting - and (iii) the environment chooses the other one. In all other cases, facilitated by the environment, the connector enacts the superior data-flow.

A connector can propagate priorities downstream (i.e., in the direction of dataflow), upstream, or in both directions.

The problem of formalizing propagation of priorities is perhaps best studied in terms of concrete connectors. To this end, the presentation of Reo so far is extended, as follows. First, Figure 7 shows four new foundational primitives that 
start (Sync $!>$ and Sync $<$ !) and end (Sync) and Sync $($ ) propagation of priorities. Second, the informal semantics of every primitive in Figure 2 is extended with:

"It always propagates others' priorities downstream and upstream, but never its own."

\section{Solution}

Idea. The idea is to decompose the abstract concept of propagation of priorities into two more concrete auxiliary metadata-flows: one from a connector to the environment and one from the environment to the connector. Through the former, called propagation metadata-flow, a connector informs its environment on which shared vertices the environment must perform reciprocal writes and takes to facilitate the propagated priority of the connector; through the latter, called conflict metadata-flow, the environment informs the connector on which shared vertices it cannot perform reciprocal writes and takes, due to conflicting propagated priorities. The direction of metadata-flows is completely independent of the direction of data-flows: metadata can flow both upstream and downstream, whereas data can flow only downstream.

Now, the plan is to model metadata-flows using colors. The problem is that metadata-flows conceptually precede normal data-flows (i.e., they happen between clock ticks), which cannot be directly modeled in the connector coloring framework (i.e., the framework only models what happens at clock ticks). The solution is to conflate metadata-flows and normal data-flows.

To model propagation metadata-flows from a connector to the environment, I introduce three new activeness colors: 4, 5, 6 (Fig. (4). In a coloring, entry $v \mapsto 4$ $(v \mapsto 5)$ models that vertex $v$ is active in the current data-flow, and was active in the preceding propagation metadata-flow downstream (upstream). To model metadata-flows from the environment to the connector, I introduce two new passiveness colors: 7,8 (Fig. 4). In a coloring, entry $v \mapsto 7(v \mapsto 8)$ models that vertex $v$ is passive in the current data flow, but was active in the preceding conflict metadata-flow downstream (upstream); this means the environment cannot write (take) through $v$, because of conflicting priorities upstream (downstream). Thus, the new instantiation of the connector coloring framework has eight colors: $\{1,2,3,4,5,6,7,8\}$.

Priority primitives. Figure 8 shows the eight-color semantics of the new, priority primitives. Coloring $\gamma_{1}$ of Sync!> models a data-flow from $v_{1}$ to $v_{2}$, preceded by a propagation metadata-flow downstream from $v_{2}$ (into the environment). Through this metadata-flow, Sync!> informs the environment that it must perform a reciprocal take on $v_{2}$. Coloring $\gamma_{2}$ is similar to $\gamma_{1}$, except that the metadata-flow does not start at $v_{2}$, but further upstream; the metadata simply flows from $v_{1}$ to $v_{2}$. Coloring $\gamma_{3}$ is similar to $\gamma_{1}$, but beside modeling a propagation metadata-flow downstream from $v_{2}$ (into the environment), it also models a propagation metadata-flow upstream from $v_{2}$ to $v_{1}$. Coloring $\gamma_{4}$ combines $\gamma_{2}$ 


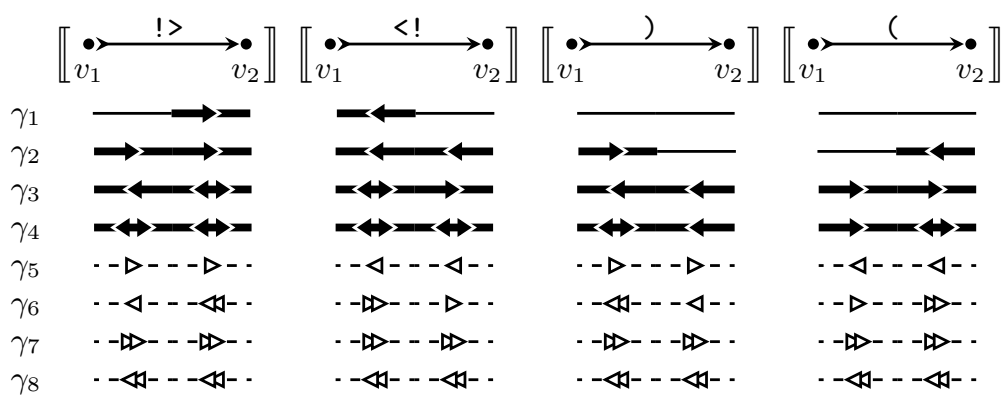

Fig. 8: Eight-color semantics of priority primitives

and $\gamma_{3}$. Colorings $\gamma_{5}-\gamma_{8}$ all model idling. Specifically, $\gamma_{5}$ and $\gamma_{7}$ permit idling if the environment cannot write through $v_{1}$, while $\gamma_{6}$ and $\gamma_{8}$ permit idling if the environment cannot take through $v_{2}$ because of conflicting propagated priorities. Note that there is no coloring that permits idling if the environment cannot take through $v_{2}$, not because of conflicting propagated priorities. The colorings of Sync $<$ are symmetric.

The key colorings of Sync) are $\gamma_{2}, \gamma_{3}$, and $\gamma_{6}$. Coloring $\gamma_{2}$ models a data-flow from $v_{1}$ to $v_{2}$, preceded by a propagation metadata-flow downstream to $v_{1}$, but no further. In this way, Sync) blocks propagation of priorities downstream. Coloring $\gamma_{3}$ models a data-flow from $v_{1}$ to $v_{2}$, preceded by a propagation metadata-flow upstream from $v_{2}$ to $v_{1}$. This shows that the blockade works only in one direction. Coloring $\gamma_{6}$ models idling, supposedly caused by conflicting propagated priorities. However, such a conflict does not really exist: Sync) only pretends it has a conflict, to enable anyone further downstream to truly ignore priorities propagated through $v_{1}$, as part of its blockade. The colorings of Sync ( are symmetric.

Common primitives. Figure 9 shows the eight-color semantics of the existing, common primitives (unary and binary); a " $+\mathrm{M}$ " annotation below a coloring means that the "horizontally mirrored" version of that coloring is part of the semantics as well. I highlight two salient aspects. First, the three-color semantics of every primitive CCA07Cos10 is strictly contained in its eight-color semantics (cf. the three-color semantics of ExclDrain and LossySync in Fig. 6). Second, coloring $\gamma_{4}$ of ExclDrain is a premier example of a propagation metadata-flow (from connector to environment) that induces a conflict metadata-flow (from environment to connector).

Figure 9 shows the eight-color semantics of the existing, common primitives (ternary). Again, the eight-color semantics strictly contain the three-color semantics. The interesting colorings are $\gamma_{6}, \gamma_{16}$, and $\gamma_{12}-\gamma_{14}$ of Merger. Coloring $\gamma_{6}$ and $\gamma_{16}$ are similar to coloring $\gamma_{4}$ of ExclDrain. Colorings $\gamma_{12}-\gamma_{14}$ are notable, because they model propagation metadata-flows, but no conflict metadata-flows, in contrast to colorings $\gamma_{6}$ and $\gamma_{16}$. This is because propagation metadata-flows upstream have no bearing on the choices made by Merger: regardless of whether 


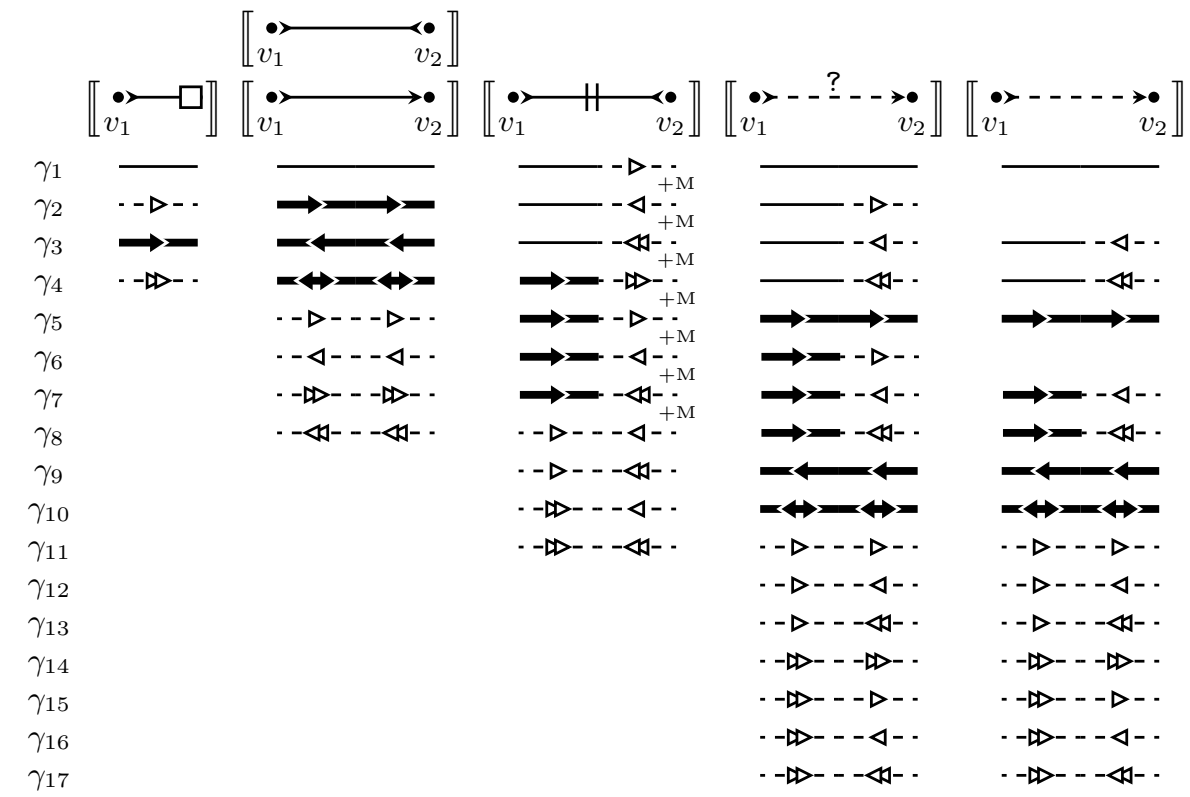

Fig. 9: Eight-color semantics of common unary and binary primitives

Merger chooses one of $\gamma_{12}-\gamma_{14}$, or one of their "vertically mirrored" versions, shared vertex $v_{3}$ is always active; this is all the propagated priority needs.

Next, to evaluate whether the eight-color semantics of the primitives compose as expected, I state and prove a number of eight-color semantics equalities.

\section{Evaluation}

Basic properties of common primitives. The following four propositions state that the common binary primitives in Fig. 2 (except LossySync) can be constructed out of unary and ternary primitives ${ }^{6}$

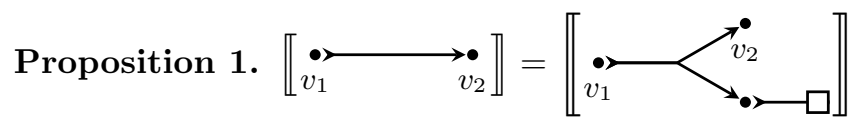

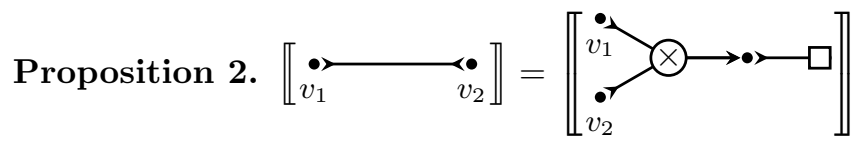

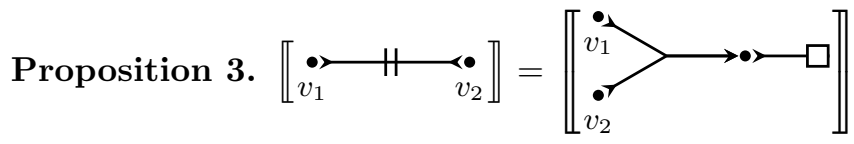

\footnotetext{
${ }^{6}$ All propositions in this paper should be interpreted modulo application of an hide operator, to remove internal vertices from the domains of colorings. This is straightforward to explicitly formalize.
} 


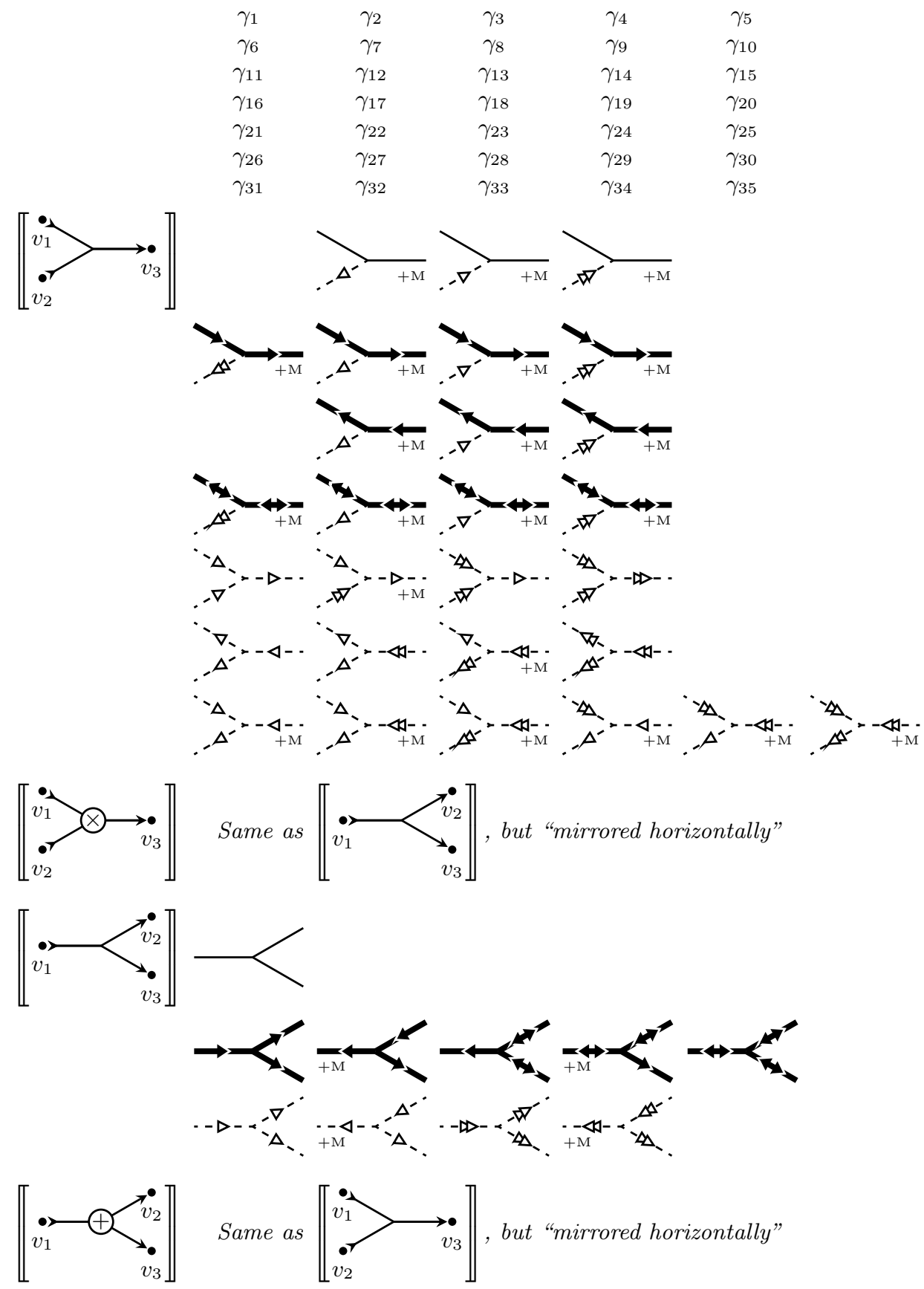

Fig. 10: Eight-color semantics of common ternary primitives 


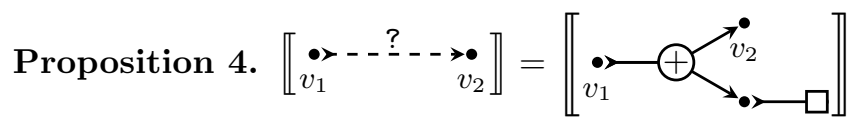

The following proposition states that LossySync? and LossySync behave differently when composed with Drain. Specifically, according to its eight-color semantics, LossySync? can (nondeterministically choose to) lose data before it reaches Drain, which LossySync cannot. This difference in semantics is intended: LossySync prioritizes not-losing over losing, whereas LossySync? does not.

\section{Proposition 5.}

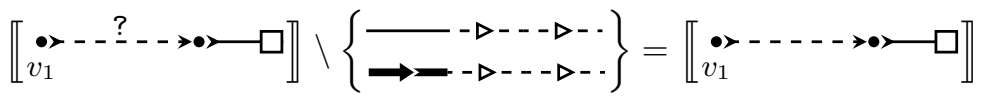

Basic properties of priority primitives. The following two propositions state that Sync!> and Sync $<$ ! and Sync) and Sync, commute. Both compounds have the same data-flows and priorities as Sync in Fig. 22 But, the former compound always propagates priorities downstream and upstream, whereas the latter compound connector, in contrast, never propagates priorities downstream or upstream.

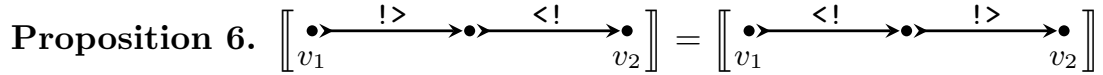

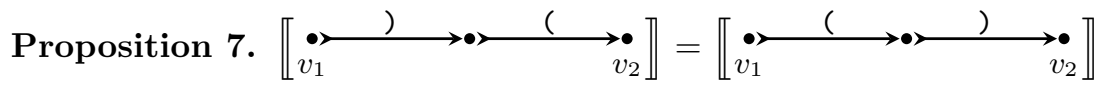

The following proposition states that Sync) is not the "inverse" of Sync!>: starting and ending propagation of priorities is not "neutral". The reason is that Sync) ends the downstream propagation of all priorities; not just those of Sync!>.

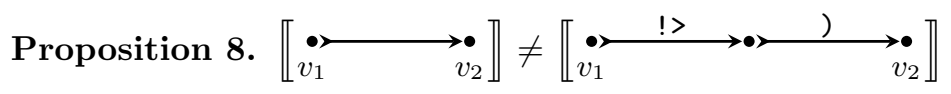

Imagine a variant of ExclDrain that, informally, has the same data-flows and priorities as ExclDrain in Fig. 2, but additionally prioritizes (1) over (2). The following proposition states that this connector, called ExcIDrain! in Fig. 11, can be constructed out of Sync!> and ExclDrain.

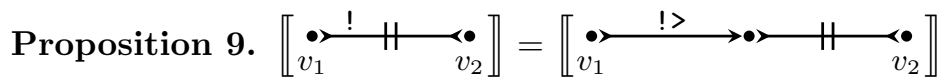

The following proposition states that conflicting propagated priorities "cancel out": the composition of ExclDrain! and Sync <! is almost the same as ExclDrain. The only difference is that the compound is saturated: the extra coloring (cf. ExclDrain) means that the compound can always ignore propagated priorities, by pretending there is a conflict. As a result, it is actually impossible to (re)construct ExclDrain! from the compound.

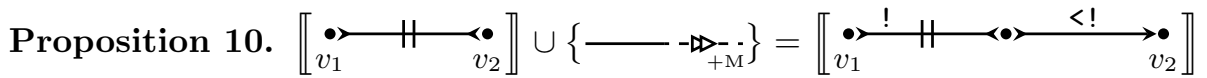




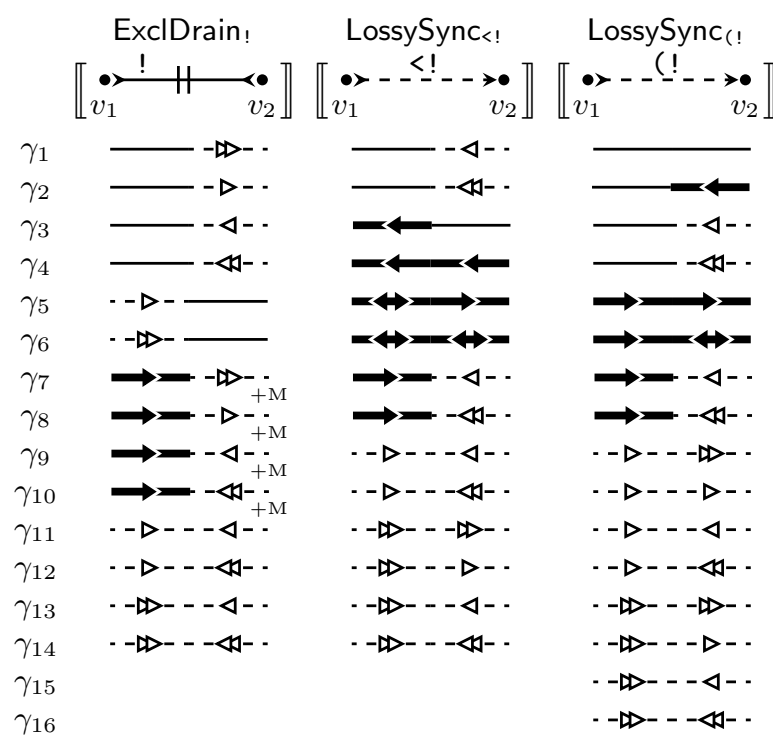

Fig. 11: Eight-color semantics of additional priority primitives

Advanced properties: context-sensitivity. Perhaps the litmus test for any formalization of propagation of priority is the construction of the context-sensitive LossySync out of the nondeterministic LossySync? and the priority primitives.

The construction proceeds in three steps. First, compose LossySync? and Sync $<$ ! The idea is that, through propagation of its own priorities, the latter forces the former to prioritize not-losing over losing. This works, but there is an undesirable side effect: the compound connector, called LossySync $<$ ! in Fig. 11, propagates its own priorities upstream, which LossySync? does not. To solve this, second, compose Sync, and LossySync $<$ ! The idea is that the former blocks the upstream propagation of LossySync <!'s priorities. This works, but there is again an undesirable side effect: the compound connector, called LossySync (! in Fig. 11 . blocks the upstream propagation of all priorities (cf. Prop. 15). To solve this, finally, compose LossySync(! with ExclRouter and Merger. The idea is that the upstream propagation of others' priorities is not blocked, essentially because the propagation can proceed via a different upstream path through the graph.

The following propositions state that using the eight-color semantics, this construction roughly works: the only discrepancy is the presence of two colorings in the eight-color semantics of the final compound - absent in the eight-color semantics of LossySync - that model partial metadata-flows. This is an interesting phenomenon: relative to the informal semantics, the colorings are not wrong. They essentially mean that it is not really necessary to propagate priorities upstream, if a data-flow from vertex $v_{1}$ to vertex $v_{2}$ is already possible without such propagation. Through the construction of LossySync, this property "incidentally" emerges. I conjecture that if this property is consistently included 
in the eight-color semantics of all primitives, including LossySync, the resulting formalization of propagation of priority fully passes this litmus test.

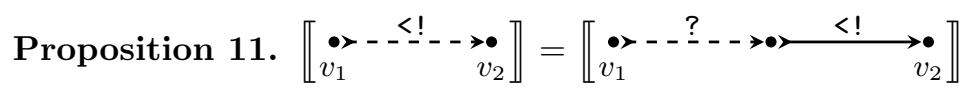

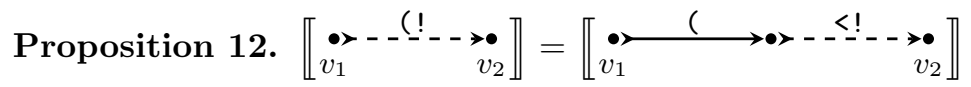

Proposition 13.

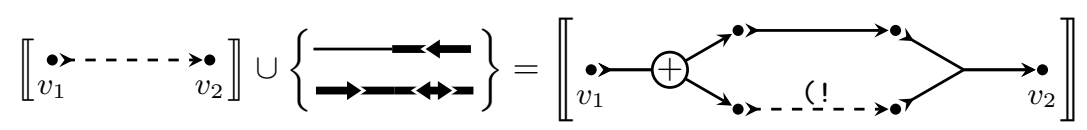

Advanced properties: ranks. Imagine a variant of Merger that, informally, has the same data-flows and priorities as Merger in Fig. 2, but additionally prioritizes (1) over (2). The following proposition states that this primitive, called Merger !>, can be composed out of Sync!> and Merger (cf. Prop. 8).

Proposition 14.

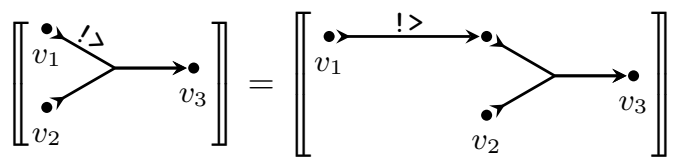

Imagine a variant of Merger with three sources instead of two. Informally, it has a data-flow from each of its sources to its targets, one of which it prioritizes over the other two. The following proposition states that this primitive, called Merger $3_{!>}$, can be composed out of Merger!> and Merger.

Proposition 15.

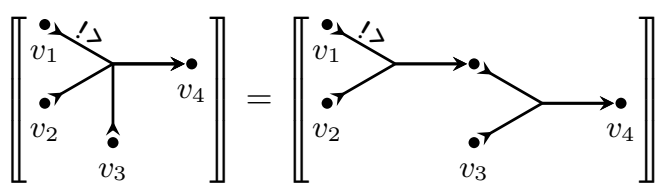

Imagine a variant of Merger $3_{\text {!> }}$ with three sources instead of two. Informally, it has a data-flow from each of its sources to its targets, one of which it prioritizes over the other two (rank \#1), and one of those two (rank \#2) of which it prioritizes over the other one (rank \#3). The following proposition states that this primitive, called Merger $3_{\text {!! >, !> }}$, can be composed out of Merger!> and Merger!>.

Proposition 16.

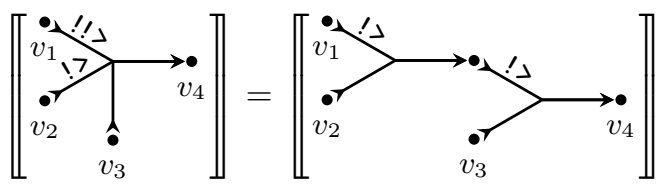




\section{Discussion}

I conclude this paper with some open issues and future work. Section 5 revealed already one open issue, namely the minor discrepancy between LossySync the primitive and LossySync the compound.

A second issue with the current formalization is exemplified by the connector in Fig. $1 \mathrm{~b}$ the eight-color semantics of this compound contains only one coloring that models idling, and moreover, this coloring has a causality loop (i.e., it is non-constructive, in Costa's sense [Cos10]). This problem is surprisingly difficult to solve in a proper way; the obvious solution (adding coloring $\left\{v_{1} \mapsto 3, v_{2} \mapsto\right.$ $\left.\left.3, v_{3} \mapsto 3\right\}\right)$ has quite adverse side effects. Perhaps the problem can be solved by adding one or more colors.

The eight-color semantics of the connector in Fig. 1c allows for a nondeterministic choice between an "upper" data-flow (from $v_{1}$ to $v_{3}$ ) and a "lower" data-flow (from $v_{1}$ to $v_{4}$ and $v_{3}$ ), because Sync!>'s priorities are propagated only downstream, not affecting the nondeterministic choice of ExclRouter, upstream. This is a reasonable interpretation of the informal semantics. An alternative interpretation, and arguably equally reasonable, is that Merger should propagate priorities from $v_{5}$ not only to $v_{3}$ but also to $v_{6}$, reversing the direction of propagation from downstream to upstream. Under this interpretation, the nondeterministic choice of ExclRouter is affected by Sync!>'s priorities, and the lower data-flow should never be chosen. It would be interesting to investigate how to model this alternative interpretation in the connector coloring framework.

Finally, the eight-color semantics of primitives and compounds quickly become prohibitively large. This makes manually reasoning about these semantics quite challenging. The development of software tooling to automate the composition of sets of colorings is imperative to continue this line of research.

\section{References}

Arb04. Farhad Arbab. Reo: a channel-based coordination model for component composition. Mathematical Structures in Computer Science, 14(3):329-366, 2004.

Arb11. Farhad Arbab. Puff, the magic protocol. In Formal Modeling: Actors, Open Systems, Biological Systems, volume 7000 of Lecture Notes in Computer Science, pages 169-206. Springer, 2011.

CCA07. Dave Clarke, David Costa, and Farhad Arbab. Connector colouring I: synchronisation and context dependency. Sci. Comput. Program., 66(3):205-225, 2007.

Cos10. David Costa. Formal Models for Component Connectors. PhD thesis, Vrije Universiteit, 2010.

CP12. Dave Clarke and José Proença. Partial connector colouring. In COORDINATION, volume 7274 of Lecture Notes in Computer Science, pages 59-73. Springer, 2012.

JKA11. Sung-Shik T. Q. Jongmans, Christian Krause, and Farhad Arbab. Encoding context-sensitivity in reo into non-context-sensitive semantic models. In $\mathrm{CO}$ ORDINATION, volume 6721 of Lecture Notes in Computer Science, pages 31-48. Springer, 2011. 
Jon18. Sung-Shik Jongmans. Formalizing Propagation of Priorities in Reo, using Eight Colors (Technical Report). Technical report, 2018. www.arxiv.org.

\section{A Definitions}

Definition 1 (Structure). $\mathbb{V}$ is the set of all vertices. $\mathbb{T}$ is the set of all types. The structure of a connector is a tuple $g=(V, E)$, where $V \subseteq \mathbb{V}$ and $E \subseteq$ $\left(2^{V} \times \mathbb{T} \times 2^{V}\right) \backslash\{(\emptyset, t, \emptyset) \mid t \in \mathbb{T}\} . \mathbb{G}$ is the set of all structures.

Definition 2 (Structural composition). S, T : $2^{\left(2^{\mathrm{V}} \times \mathbb{T} \times 2^{\mathrm{V}}\right)} \rightarrow 2^{\mathbb{V}}$ are the functions defined by the following equations:

$$
\begin{aligned}
& \mathrm{S}(E)=\bigcup\left\{V \mid\left(V, t, V^{\prime}\right) \in E\right\} \backslash \bigcup\left\{V^{\prime} \mid\left(V, t, V^{\prime}\right) \in E\right\} \\
& \mathrm{T}(E)=\bigcup\left\{V^{\prime} \mid\left(V, t, V^{\prime}\right) \in E\right\} \backslash \bigcup\left\{V \mid\left(V, t, V^{\prime}\right) \in E\right\}
\end{aligned}
$$

$\bowtie: \mathbb{G} \times \mathbb{G} \rightarrow \mathbb{G}$ is the partial operation defined by the following equation:

$$
\left(V_{1}, E_{1}\right) \bowtie\left(V_{2}, E_{2}\right)= \begin{cases}\left(V_{1} \cup V_{2}, E_{1} \cup E_{2}\right) & \text { if: } \mathrm{S}\left(E_{1}\right) \cap \mathrm{T}\left(E_{2}\right)=\mathrm{S}\left(E_{2}\right) \cap \mathrm{T}\left(E_{1}\right) \\ \perp & \text { otherwise }\end{cases}
$$

Definition 3 (Behavior). $\mathbb{C}$ is the set of all colors. A coloring $\gamma$ over $V$ is a function from $V$ to $\mathbb{C}$. $\mathbb{C o L}(V)=V \rightarrow \mathbb{C}$ is the set of all colorings over $V$. The behavior of a connector $(V, E)$ is a set $\Gamma \subseteq \mathbb{C O L}(V)$ of colorings.

Definition 4 (Behavioral composition).

$\bowtie:\left(\mathbb{C O L}\left(V_{1}\right) \times \mathbb{C O L}\left(V_{2}\right) \rightarrow \mathbb{C O L}\left(V_{1} \cup V_{2}\right)\right) \cup\left(2^{\operatorname{CoL}\left(V_{1}\right)} \times 2^{\operatorname{CoL}\left(V_{2}\right)} \rightarrow 2^{\mathbb{C o L}\left(V_{1} \cup V_{2}\right)}\right)$ is the partial function defined by the following equations:

$$
\begin{aligned}
\gamma_{1} \bowtie \gamma_{2} & = \begin{cases}\gamma_{1} \cup \gamma_{2} & \text { if: } \gamma_{1}(p)=\gamma_{2}(p) \text { for-all } p \in \operatorname{dom}\left(\gamma_{1}\right) \cap \operatorname{dom}\left(\gamma_{2}\right) \\
\perp & \text { otherwise }\end{cases} \\
\Gamma_{1} \bowtie \Gamma_{2} & =\left\{\gamma_{1} \bowtie \gamma_{2} \mid \gamma_{1} \in \Gamma_{1} \text { and } \gamma_{2} \in \Gamma_{2} \text { and } \gamma_{1} \bowtie \gamma_{2} \in \operatorname{dom}(\bowtie)\right\}
\end{aligned}
$$

Definition 5 (Denotation). With $\mathcal{T}: \mathbb{T} \rightarrow\left(2^{\mathbb{V}} \times 2^{\mathbb{V}}\right) \rightarrow \bigcup\left\{2^{\operatorname{CoL}(V)} \mid V \subseteq \mathbb{V}\right\}$, $\llbracket \cdot \rrbracket: \mathbb{G} \rightarrow \bigcup\{\mathbb{C O L}(V) \mid V \subseteq \mathbb{V}\}$ is the function defined by the following equation:

$$
\llbracket(V, E) \rrbracket=\bowtie\left\{\mathcal{T}(t)\left(V, V^{\prime}\right) \mid\left(V, t, V^{\prime}\right) \in E\right\}
$$

Theorem 1. $\llbracket g_{1} \bowtie g_{2} \rrbracket=\llbracket g_{1} \rrbracket \bowtie \llbracket g_{2} \rrbracket$ 